\title{
Resident Fibroblast MKL1 Is Sufficient to Drive Pro-fibrogenic Response in Mice
}

OPEN ACCESS

Edited by:

Cheng Yang,

Fudan University, China

Reviewed by:

Fernando Rodriguez-Pascual, Spanish National Research Council

(CSIC), Spain

Katalin Szaszi,

St. Michael's Hospital, Canada

*Correspondence:

Qianwen Zhao

591378701@qq.com

Tianfa Li

litf79997@163.com

Xianhua Gui

xianxian.xian@163.com

Specialty section:

This article was submitted to Molecular and Cellular Pathology,

a section of the journal

Frontiers in Cell and Developmental

Biology

Received: 10 November 2021 Accepted: 23 December 2021

Published: 01 February 2022

Citation:

Huang S, Shao T, Liu H, LiT, GuiX and Zhao Q (2022) Resident Fibroblast MKL1 Is Sufficient to Drive Profibrogenic Response in Mice. Front. Cell Dev. Biol. 9:812748. doi: 10.3389/fcell.2021.812748

\author{
Shan Huang ${ }^{1,2}$, Tinghui Shao ${ }^{1}$, Hong Liu $^{1}$, Tianfa $L i^{1,2 *}$, Xianhua Gui ${ }^{3 *}$ and Qianwen Zhao ${ }^{1,2 *}$ \\ ${ }^{1}$ Key Laboratory of Targeted Intervention of Cardiovascular Disease and Collaborative Innovation Center for Cardiovascular \\ Translational Medicine, Department of Pathophysiology, Nanjing Medical University, Nanjing, China, ${ }^{2}$ Hainan Provincial Key \\ Laboratory for Tropical Cardiovascular Diseases Research, Key Laboratory of Emergency and Trauma of Ministry of Education, \\ Department of Cardiology, Research Unit of Island Emergency Medicine of Chinese Academy of Medical Sciences, The First \\ Affiliated Hospital of Hainan Medical University, Haikou, China, ${ }^{3}$ Department of Respiratory Medicine, Affiliated Nanjing Drum \\ Tower Hospital, Nanjing University School of Medicine, Nanjing, China
}

Fibrosis is an evolutionarily conserved pathophysiological process serving bifurcated purposes. On the one hand, fibrosis is essential for wound healing and contributes to the preservation of organ function. On the other hand, aberrant fibrogenic response may lead to tissue remodeling and precipitate organ failure. Recently lineage tracing studies have shown that resident fibroblasts are the primary mediator of fibrosis taking place in key organs such as the heart, the lungs, and the kidneys. Megakaryocytic leukemia 1 (MKL1) is transcriptional regulator involved in tissue fibrosis. Here we generated resident fibroblast conditional MKL1 knockout (CKO) mice by crossing the $M k / 1^{\mathrm{f} / \mathrm{f}}$ mice to the Col1a2-Cre ERT2 mice. Models of cardiac fibrosis, pulmonary fibrosis, and renal fibrosis were reproduced in the CKO mice and wild type (WT) littermates. Compared to the WT mice, the CKO mice displayed across-the-board attenuation of fibrosis in different models. Our data cement the pivotal role MKL1 plays in tissue fibrosis but point to the cellular origin from which MKL1 exerts its pro-fibrogenic effects.

Keywords: fibrosis, fibroblast, transcription factor, wound healing, MKL1 (MRTF-A)

\section{INTRODUCTION}

Fibrosis is considered a key part of the wound healing process that safeguards the architectural and functional integrity of the host (Henderson et al., 2020). On the one hand, fibrotic tissue (scar) helps cover the wound to prevent excessive loss of blood and maintain body fluid homeostasis. On the other hand, accelerated production of extracellular matrix (ECM) proteins contributes to interstitial remodeling and preservation of organ function. In order to fulfill these requirements, a specialized cell type emerging from the injured tissues, termed "myofibroblast", is able to both perform muscle-like contraction and synthesize ECM proteins (Pakshir et al., 2020). Spatiotemporally controlled activation and deactivation of myofibroblasts, acting as the chief effector of fibrosis, ensures proper initiation and termination of the fibrogenic response. Dysregulation of myofibroblast maturation and resolution, on the contrary, is invariably associated with the loss of key organ function. For instance, insufficient activation of myofibroblasts in the aging heart triggers defective wound healing and heart failure following myocardial infarction (Bujak et al., 2008). Alternatively, depletion of excessive myofibroblasts mitigates adverse interstitial remodeling and improves organ function in mice (Kaur et al., 2016; Meng et al., 2016; Xu et al., 2020). 
Megakaryocytic leukemia 1 (MKL1) is a transcriptional regulator initially identified and characterized by Eric Olson and colleagues as a co-factor for serum response factor (SRF) to regulate the transcription of contractile genes (Wang et al., 2002). Early studies have found that over-expression of MKL1 is sufficient to stimulate the transcription of contractile genes in non-muscle cells indicating that MKL1 is the rate-limiting factor, at least under certain circumstances, in the acquisition of a contractile phenotype (Zhang et al., 2007). On the contrary, global MKL1 knockout (KO) mice are indistinguishable from their wild type (WT) littermates in terms of smooth muscle contraction or contractile gene expression in physiological settings (Li et al., 2006; Sun et al., 2006), which again seems to allude to the notion that MKL1 is required for the acquisition, rather than the maintenance, of a contractile phenotype. Indeed, research in the past decade has provided mounting evidence that MKL1 is a master regulator in tissue fibrosis. Small et al. have presented the first in vivo evidence that the global MKL1 KO mice display significantly weakened cardiac fibrosis compared to the WT mice in a model of myocardial infarction (Small et al., 2010). This report was followed by a string of studies that implicate MKL1 in the regulation of pulmonary fibrosis (Zhou et al., 2013), scleroderma (Shiwen et al., 2015), liver fibrosis (Fan et al., 2015; Tian et al., 2015; Wu X. et al., 2020), renal fibrosis (Xu et al., 2015; Mao et al., 2020b), and intestinal fibrosis (Johnson et al., 2014). Despite these observations, it is not clear from which cell type MKL1 exerts its pro-fibrogenic effects due to its universal expression pattern (Olson and Nordheim, 2010). For instance, Weng et al. have previously that mice with endothelial cellspecific MKL1 deletion are partially resistant to angiotensin II (Ang II) induced cardiac fibrosis and heart failure (Weng et al., 2015). Similarly, Liu et al. have recently reported that conditional deletion of MKL1 from the myeloid lineage protects the mice from pressure overload induced pathological hypertrophy and cardiac fibrosis in mice (Liu et al., 2021a).

Recent advances in genetic lineage tracing technique have greatly facilitated the attempt to uncover the origin(s) of myofibroblasts in tissue fibrosis. Although substantial variations exist in the accumulated data depending on both the type of tissue examined and the method used to induce fibrosis, there appears to be a consensus that resident fibroblasts represent a predominant source for myofibroblast in the heart (Kanisicak et al., 2016), in the kidneys (LeBleu et al., 2013), and in the lungs (Zepp et al., 2017). Based on these results, we hypothesized that MKL1 deficiency in tissue resident fibroblasts may be sufficient to influence fibrosis. Our data demonstrate that conditional MKL1 deletion in resident fibroblasts attenuates myocardial fibrosis, renal fibrosis, and pulmonary fibrosis in vivo.

\section{METHODS}

\section{Animals}

All animal protocols were reviewed and approved the intramural Ethics Committee on Humane Treatment of Laboratory Animals of Nanjing Medical University. The mice were maintained in an
SPF environment with $12 \mathrm{~h}$ light/dark cycles and libitum access to food and water. Resident fibroblast specific MKL1 knockout mice were created by crossing by the $M k l 1^{\mathrm{f} / \mathrm{f}}$ mice (Liu et al., 2021a; Liu et al., 2021b) with the Col1a2-Cre ${ }^{\text {ERT2 }}$ mice (Wang et al., 2016). Cardiac fibrosis was induced by permanent ligation of left anterior descending coronary artery as previously described (Yang et al., 2017). Renal fibrosis was induced by the unilateral ureteral obstruction procedure as previously described (Mao et al., 2020a; Mao et al., 2020b). Pulmonary fibrosis was induced by intratracheal instillation of bleomycin as previously described (Xu et al., 2011; Ianni et al., 2021).

\section{Bronchoalveolar Lavage Fluid}

7 days following intratracheal bleomycin administration or $0.9 \%$ saline treatment, BALF was performed. The mice were sacrificed in deep anesthesia by atlanto-occipital dislocation. The trachea was exposed and a catheter was used to intubate the mice. The lungs were instilled 3 times using $1 \mathrm{ml}$ of $0.9 \%$ saline. The BALF was collected, centrifuged at $400 \mathrm{~g}$ for $10 \mathrm{~min}$, the total cells in the pellet were collected for detecting RNA levels, while BALF was preserved at $-20^{\circ} \mathrm{C}$.

\section{Urine Analyses}

Urine samples were collected from the mice after 14-days UUO surgery. Urinary creatinine $(\mathrm{Cr})$ and urea nitrogen (BUN) were quantified separately using commercial assay kits from Nanjing Jiancheng Bioengineering Institute (Nanjing, China) according to the manufacturer's instructions.

\section{Cardiac Function Assessment by Echocardiography}

A non-invasive transthoracic echocardiographic examination was performed at the 21st day after ligation of left anterior descending coronary artery by using a Vevo 2,100 (Visual sonics, Canada), equipped with a $30-\mathrm{MHz}$ transducer. The mice were anesthetized by $2 \%$ isoflurane gas $\left(1.5 \%\right.$ with $\mathrm{O}_{2}$ $1 \mathrm{~L} / \mathrm{min}$ ). Two-dimensional guide $\mathrm{M}$-mode tracings were recorded, and the ejection fraction (EF), fractional shortening (FS), late diastolic transmitral flow velocity $\left(\mathrm{E}^{\prime} / \mathrm{A}^{\prime}\right)$ and early diastolic mitral annular velocity $\left(E / E^{\prime}\right)$ were measured or further calculated.

\section{RNA Isolation and Real-Time Quantitative Polymerase Chain Reaction (RT-qPCR) Analysis}

RNeasy RNA isolation kit (Qiagen) was used to extract total RNA from tissue sample of mice according to the manufacturer's procedure. The extracted RNA was dissolved in diethypyrocarbonate (DEPC)-treated water, and the RNA concentration was determined by optical density measurement at $260 \mathrm{~nm}$ using a spectrophotometer. RNA sample $(1 \mu \mathrm{g})$ was subsequently reverse transcribed using a HiScript III RT SuperMix (Vazyme). Real-time PCR reactions were carried out on an ABI Prism StepOne Plus system with a commercial SYBR green kit (Vazyme). The primers used were as follows: 18s: $5^{\prime}$ - 
GGACCAGAG CGAAAGCATTTGCC-3' (forward) and 5' -TCA ATCTCGGGTGGCTGA ACGC-3' (reverse); Acta2: $5^{\prime}$-CCT GTTTCGGGAGCAGAA-3' (forward) and 5'-GGTTATATA GCCCCCTGG-3' (reverse); Colla1: $5^{\prime}$-GCTCCTCTTAGG GGCCACT-3' (forward) and 5' ${ }^{\prime}$-CCACGTCTC ACCATTGGGG -3' (reverse); Col3al: 5' ${ }^{\prime}$ TAGTAACATGGA AACCTGGGGAAA-3' (forward) and 5'-CCATAGCTGAAC TGAAAACCACC-3' (reverse). Ct values of target genes were normalized to the $\mathrm{Ct}$ values of the endogenous control $18 \mathrm{~s}$ using the $\Delta \Delta \mathrm{Ct}$ method and expressed as relative mRNA expression levels compared to the control group which is arbitrarily set as 1 .

\section{Western Blot Analysis}

The total protein was extracted from the samples using radioimmunoprecipitation assay (RIPA) buffer $(50 \mathrm{mM}$ Tris pH7.4, $150 \mathrm{mM} \mathrm{NaCl}, 1 \%$ Triton X-100) with freshly added protease inhibitor tablet (Roche). Protein concentrations were quantified using a Bicinchoninic Acid Protein Assay kit (Biosky Biotechnology, Nanjing, China) according to the manufacturer's protocol. Thirty micrograms of protein samples were separated by $8 \%$ sodium dodecyl sulfate-polyacrylamide gel electrophoresis (SDS-PAGE) and electrophoretically transferred to PVDF membranes $(0.45 \mu \mathrm{m}$; EMD Millipore, Billerica, MA, United States). Membranes were blocked for $1 \mathrm{~h}$ at room temperature with 5\% BSA (Sangon Biotech Co., Ltd.) in TBSTween-20 (containing $5 \mathrm{mM}$ Tris- $\mathrm{HCl}(\mathrm{pH} 7.6), 136 \mathrm{mM} \mathrm{NaCl}$ and $0.05 \%$ Tween-20). Then Membranes were incubated with anti-TBP (Proteintech, China, 22006-1-AP, 1:1,000), anti-MKL1 (Proteintech, China, 21166-1-AP, 1:1,000) overnight at $4^{\circ} \mathrm{C}$. Subsequently, the primary antibodies were detected with secondary antibody (anti-Rabbit, Beyotime Institute of Biotechnology, China, A0208, 1:3,000) and scanned by using enhanced chemiluminescence western detection reagents (Thermo Fisher Scientific, Inc.). Image-Lab version 5.2.1 software (Bio-Rad Laboratories, Inc.) was used to quantify the intensity of the band in western blot assay.

\section{Masson, Sirius Red Staining and Hematoxylin/Eosin Staining}

Histological analysis was performed with conventional methods as previously described (Li et al., 2018). Briefly, tissue samples were placed in $4 \%$ paraformaldehyde (PFA) in PBS overnight and then dehydrated in a gradient ethanol solution and embedded in paraffin. The tissue in the paraffin block was cut to a thickness of $5 \mu \mathrm{m}$.

HE staining was conducted according to routine protocols. Briefly, after deparaffinization and rehydration, the tissue sections were stained with hematoxylin solution for $5 \mathrm{~min}$ followed by immersed in $1 \%$ acid ethanol $(1 \% \mathrm{HCl}$ in $70 \%$ ethanol) for $15 \mathrm{~s}$, and then rinsed in tap water for $10 \mathrm{~min}$. Subsequently, the sections were stained with eosin solution for $2 \mathrm{~min}$ and followed by dehydration with graded alcohol and clearing in xylene. Pictures were taken using an Olympus IX70 microscope (Olympus, Tokyo, Japan).

PicroSirius Red staining (Sigma-Aldrich) was conducted with PicroSirius Red kit. Sections were incubated in PicroSirius Red solution for $1 \mathrm{~h}$, and then rinsed in distilled water. Subsequently, the dehydrated and mounted. Pictures were taken using an Olympus IX-70 microscope (Olympus, Tokyo, Japan) and polarizing microscope (Olympus VS200, Yokyo, Japan). The fibrotic area of each picture was analyzed by Image J software.

Masson's trichrome staining was conducted with Trichrome Stain Kit (Sigma-Aldrich). Briefly, the paraffin-embedded sections were cut and placed on standard microscopy slides. After deparaffinization and rehydration, the slides were immersed in distilled water. Subsequently, the sections were stained in weigert's hematoxylin for $5 \mathrm{~min}$, and then washed again with tap water for $5 \mathrm{~min}$ and rinsed in distilled water. Next, the slides were stained in scarlet-acid fuchsin for $5 \mathrm{~min}$, rinsed in $0.3 \%$ acetic acid, incubated in phosphomolybdic acid for $2 \mathrm{~min}$, dyed with aniline blue for $5 \mathrm{~min}$, and fixed in $0.3 \%$ acetic acid for $1 \mathrm{~min}$. Finally, the slides were dehydrated and mounted. Pictures were taken using an Olympus IX-70 microscope (Olympus, Tokyo, Japan) and the fibrotic area of each picture was analyzed by Image $J$ software.

\section{Immunofluorescence and Immunohistochemistry Staining}

For immunofluorescence staining of MKL1, FSP1 and Col1a, the tissue slices were incubated with anti-MKL1 (Abcam, 1:200), anti-FSP1 antibodies (Proteintech, 1:100) and anti- Colla (Abcam, 1:200) respectively, in one humidified chamber at $4^{\circ} \mathrm{C}$ overnight, followed by incubation with FITC-labeled secondary antibodies at $37^{\circ} \mathrm{C}$ for $1 \mathrm{~h}$. Then, cell nuclei were stained with DAPI (Sigma) for $15 \mathrm{~min}$. The immune stained images were captured using a confocal microscope (Olympus, Tokyo, Japan).

Immunohistochemistry was performed. Lung paraffinembedded sections were permeabilized and blocked with 5\% BSA, and then incubated with anti-CD68 (Proteintech, 1:100), and anti-CD45 (Proteintech, 1:100) respectively at $4^{\circ} \mathrm{C}$ overnight, then incubated with secondary antibody (Beyotime Institute of Biotechnology, 1:200) at room temperature for $1 \mathrm{~h}$. Positive immunostaining was visualized by using the diaminobenzidine substrate (DAB, Thermo Fisher, United States) for $1 \mathrm{~min}$. Next hematoxylin was utilized to stain nuclei for $20 \mathrm{~s}$. The immune stained images were captured using a confocal microscope (Olympus, Tokyo, Japan).

\section{Statistical Analysis}

All data were presented as means \pm SD. Two group comparisons were made using two-tailed Student's t tests. Multiple group comparisons were made using one-way analysis of variance. A significant difference between groups was considered as $p$ values $<0.05$.

\section{RESULTS}

\section{Validation of Megakaryocytic Leukemia 1 Deletion in Resident Fibroblasts}

In order to delete MKL1 specifically in resident fibroblasts, the $M k l 1$-flox mice were crossed to the Colla2-Cre ${ }^{\mathrm{ERT} 2}$ mice, which 

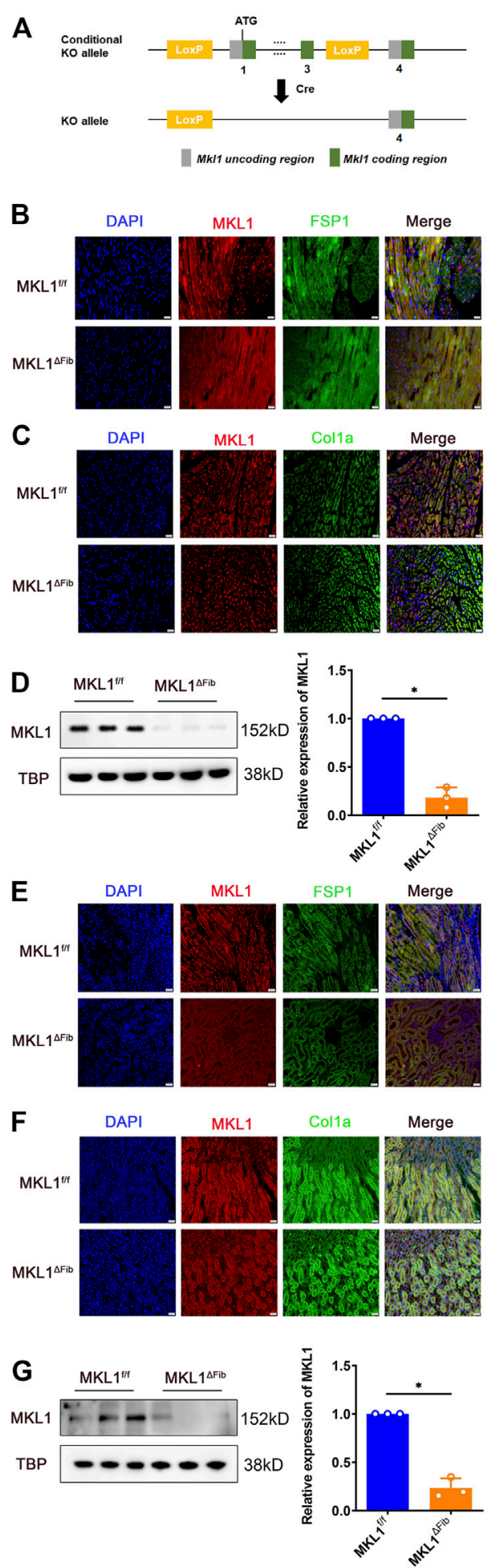

FIGURE 1 | Validation of MKL1 deletion in resident fibroblasts. WT and CKO mice were injected with tamoxifen $(50 \mathrm{mg} / \mathrm{kg}$ ) for $10 \mathrm{~d}$. (A) Scheme of protocol. (B) MKL $1^{+} \mathrm{FSP} 1^{+}$cells in cardiac tissue were measured by immunofluorescence staining. Scale bar, $20 \mu \mathrm{m}$. (C) $\mathrm{MKL}^{+}{ }^{+} \mathrm{Col} 1 \mathrm{a}^{+}$cells in cardiac tissue were measured by immunofluorescence staining. Scale bar, $20 \mu \mathrm{m}$. (D) primary cardiac fibroblasts were isolated from the MKL $1^{\Delta \mathrm{Fib}}$ heart and the $M K L 1^{f / f}$ heart, western blotting detected the MKL1 protein level. (E)

(Continued)
FIGURE 1 | Immunofluorescence staining showed the MKL1 ${ }^{+} \mathrm{FSP} 1^{+}$cells in renal tissue. Scale bar, $20 \mu \mathrm{m}$. (F) Immunofluorescence staining showed the $\mathrm{MKL} 1^{+} \mathrm{Col}_{1 \mathrm{a}^{+}}$cells in renal tissue. Scale bar, $20 \mu \mathrm{m}$. (G) Western blotting detected the MKL1 protein level in $\mathrm{MKL} 1^{\Delta \mathrm{Fib}}$ and the $\mathrm{MKL} 1^{\mathrm{f} / \mathrm{f}}$ renal resident fibroblasts. $\mathrm{N}=3$ mice. Data represent mean $\pm \mathrm{SD}$. ${ }^{*} p<0.05$, two-tailed $t$-test.

upon injection of tamoxifen allow efficient removal the floxed allele in cells that express collagen type I alpha 2 chain (Colla2) (Zheng et al., 2002) (Figure 1A). Immunofluorescence staining performed with paraffin embedded cardiac sections using an antiMKL1 antibody and an anti-FSP1 antibody showed that there were fewer double $\mathrm{MKL}^{+}{ }^{+} \mathrm{FSP} 1^{+}$cells in the $\mathrm{MKL}_{1}{ }^{\triangle \mathrm{Fib}}$ heart than in the $\mathrm{MKL1}^{\mathrm{f} / \mathrm{f}}$ heart (Figure 1B). Moreover, staining with antibodies raised against another fibroblast marker Colla2 also identified the knockout efficiency of MKL1 in $\mathrm{MKL}^{\triangle \mathrm{Fib}}$ mice. As shown in Figure 1C, MKL1 expression in fibroblasts was dramatically decreased in $\mathrm{MKL1}^{\triangle \mathrm{Fib}}$ mice, compared with $\mathrm{MKL1}^{\mathrm{f} / \mathrm{f}}$ mice. Alternatively, primary cardiac fibroblasts were isolated from the $\mathrm{MKL}^{\Delta \mathrm{Fib}}$ heart and the $\mathrm{MKL1}^{\mathrm{f} / \mathrm{f}}$ heart. Western blotting showed that MKL1 protein levels were significantly lower in the $\mathrm{MKL1}^{\triangle \mathrm{Fib}}$ cells than in the $\mathrm{MKL}^{\triangle \mathrm{Fib}}$ cells (Figure 1D). Similar experiments were performed and verified that MKL1 was specifically deleted in renal resident fibroblasts (Figures 1E-G).

\section{Megakaryocytic Leukemia 1 Deletion in Resident Fibroblasts Attenuates Cardiac Fibrosis}

We evaluated the effect of MKL1 deletion in resident fibroblasts on cardiac fibrosis in a classic model in which the left anterior descending artery was permanently ligated to induce myocardial infarction (MI, Figure 2A); significant cardiac fibrosis typically develops within 7 days of the surgical procedure (Cleutjens et al., 1995). Masson's trichrome staining (Figure 2B) and picrosirius red (Figure 2C) staining both showed significantly diminished fibrotic areas in the MKL1 ${ }^{\triangle \mathrm{Fib}}$ hearts compared to the $\mathrm{MKL1}^{\mathrm{f} / \mathrm{f}}$ hearts. In addition, collagen fiber size and alignment with picrosirius staining can be estimated under polarized light, which is correlated with collagen cross-linking and maturation (Laure Rittié, 2017). Thus, we assessed the collagen packing by visualization under polarized light. As shown in Figure 2C, the collagen fiber size, detected by polarized light, in $\mathrm{MKL1}^{\triangle \mathrm{Fib}}$ mice was significantly decreased compared to the $M K L 1^{\mathrm{f} / \mathrm{f}}$ mice. Hydroxylproline quantification confirmed that the accumulation of collagenous tissues was reduced in the $\mathrm{MKL1}^{\Delta \mathrm{Fib}}$ hearts compared to the $\mathrm{MKL1}^{\mathrm{f} / \mathrm{f}}$ hearts (Figure 2D). Quantitative PCR analysis revealed that the expression levels of several pro-fibrogenic molecules, including a-SMA (encoded by Acta2), collagen type I (encoded by Col1a1), collagen type III (encoded by Col3a1), and the lysyl oxidases (encoded by Lox) were collectively downregulated in the heart by the loss of MKL1 in fibroblasts (Figure 2E). These data showed that cardiac fibrosis after MI in $\mathrm{MKL}^{\Delta \mathrm{Fib}}$ mice were significantly attenuated, compared with that in $\mathrm{MKL1}^{\mathrm{f} / \mathrm{f}}$ mice. 

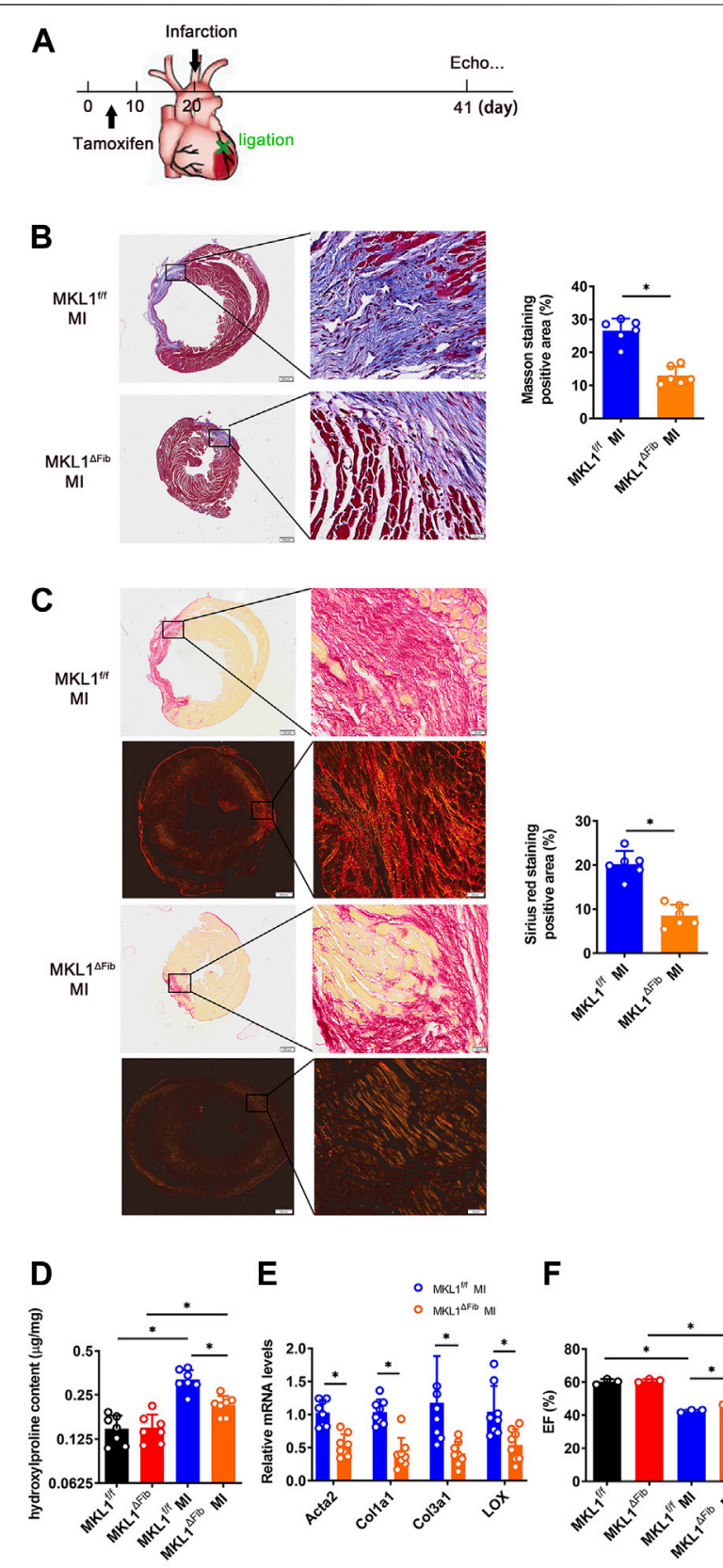

$\mathbf{F}$
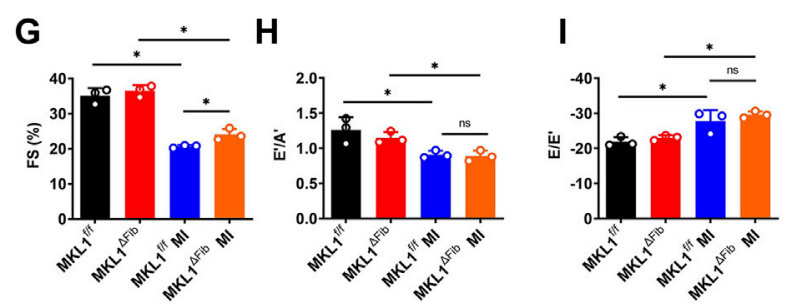

FIGURE 2 | MKL1 deletion in resident fibroblasts attenuates cardiac fibrosis. (A) Schematic diagram of myocardial infarction (MI)-induced cardiac fibrosis in $\mathrm{MKL}_{1}{ }^{\mathrm{f} / \mathrm{f}}$ and MKL1${ }^{\Delta \mathrm{Fib}}$ mice. Tamoxifen $(50 \mathrm{mg} / \mathrm{kg} / \mathrm{d})$ was injected into mice by intraperitoneal injection (i.p.) for 10 days. The mice were then subjected to Ml surgery for 21 days. (B) Masson's trichrome staining. Scale bar, $500 \mu \mathrm{m}$. N = 6 mice. (C) Picrosirius red staining under white light or (Continued)
FIGURE 2 | polarized light. Scale bar, $500 \mu$ m. $N$ = 6 mice. (D) Hydroxylproline level were detected by test kit. $N=7$ mice. (E) Pro-fibrogenic genes were measured by RT-qPCR. $N=8$ mice. (F) Ejection fraction (EF) values. $N=3$ mice. (G) Fractional shortening (FS) values. $\mathrm{N}=3$ mice. (H) Late diastolic transmitral flow velocity ( $\left.E^{\prime} / A^{\prime}\right)$ values. $N=3$ mice. (I) Early diastolic mitral annular velocity $\left(E / E^{\prime}\right)$ values. $N=3$ mice. Data represent mean $\pm S D$. ${ }^{*} p<$ 0.05 , two-tailed $t$-test.

Next, echocardiographic measurements were taken to gauge the potential impact of MKL1 deletion in resident fibroblasts on post-MI heart functions. As shown in Figure $2 \mathrm{~F}$ and Figure 2G, the $\mathrm{MKL1}^{\Delta \mathrm{Fib}}$ mice had slightly better systolic functions, measured by ejection fraction (EF) and fractional shortening (FS), than the $\mathrm{MKLl}^{\mathrm{f} / \mathrm{f}}$ mice. However, no difference in diastolic functions, as measured by late diastolic transmitral flow velocity (E'/ $\mathrm{A}^{\prime}$, Figure $2 \mathbf{H}$ ) and the ratio of mitral peak velocity of early filling to early diastolic mitral annular velocity (E/E', Figure 2I), was discernable between the $\mathrm{MKL1}^{\triangle \mathrm{Fib}}$ mice and the $\mathrm{MKL1}^{\mathrm{f} / \mathrm{f}}$ mice.

\section{Megakaryocytic Leukemia 1 Deletion in Resident Fibroblasts Attenuates Renal Fibrosis}

In order to evaluate the effect of MKL1 deletion in resident fibroblasts on renal fibrosis, the procedure of unilateral ureteral obstruction (UUO) was performed (Figure 3A); significant renal fibrosis can be detected within a week following the surgery (Klahr and Morrissey, 2002; Chevalier et al., 2009). Similar to the model of myocardial fibrosis, attenuation of renal fibrosis was detected in the $\mathrm{MKL1}^{\triangle \mathrm{Fib}}$ kidneys compared to the $\mathrm{MKL1}^{\mathrm{f} / \mathrm{f}}$ kidneys, as evidenced by Masson's trichrome staining (Figure 3B), picrosirius red staining (Figure 3C), and quantification of renal hydroxylproline levels (Figure 3D). Moreover, as shown in Figure 3C, polarized light results found the collagen fiber size in the $\mathrm{MKL1}^{\Delta \mathrm{Fib}}$ kidneys was dramatically decreased, compared with $\mathrm{MKL1}^{\mathrm{f} / \mathrm{f}}$ kidneys. Expression levels of pro-fibrogenic genes including Acta2, Colla1, Col3a1 and Lox were lower in the MKL1 ${ }^{\Delta \mathrm{Fib}}$ kidneys than the $\mathrm{MKL1}^{\mathrm{f} / \mathrm{f}}$ kidneys as revealed by qPCR (Figure 3E), confirming the obvious trend of improvement in $\mathrm{MKL1}^{\Delta \mathrm{Fib}}$ kidneys.

To determine the impact of MKL1 deletion in resident fibroblasts on renal functions, the plasma levels of urea nitrogen $(\mathrm{BUN})$ and creatinine $(\mathrm{Cr})$ were determined. As shown in Figure 3F and Figure 3G, plasmas BUN levels and Cr levels were comparable between the $\mathrm{MKL1}^{\Delta \mathrm{Fib}}$ mice and the $\mathrm{MKL}^{\mathrm{f} / \mathrm{f}}$ mice, suggesting that fibroblast resident MKL1 probably does not contribute to clearance of metabolic waste by the glomeruli.

\section{Megakaryocytic Leukemia 1 Deletion in Resident Fibroblasts Attenuates Pulmonary Fibrosis}

We next investigated the how MKL1 deletion in resident fibroblasts might alter pulmonary fibrosis. To this end, a 

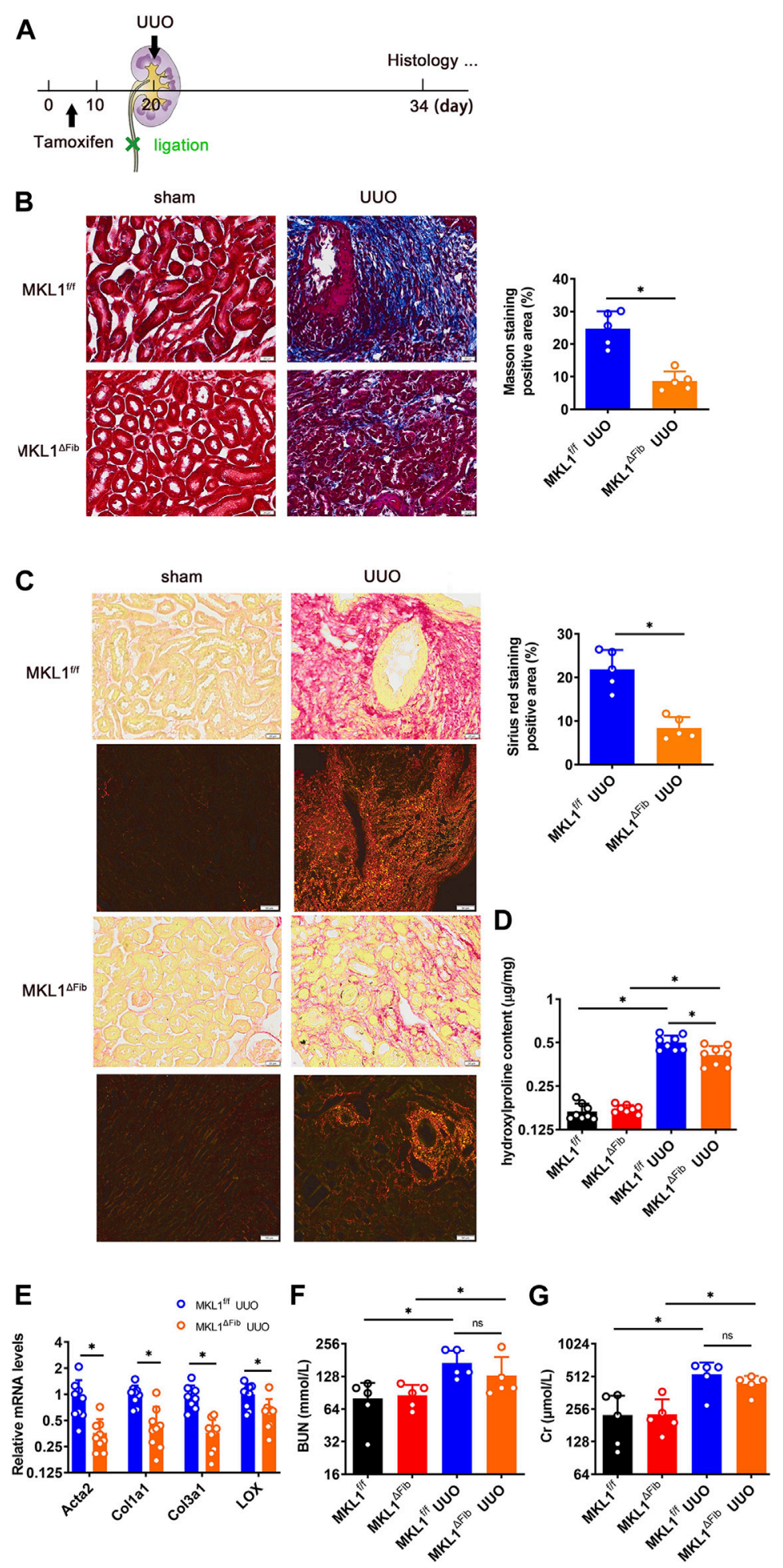

FIGURE 3 | MKL1 deletion in resident fibroblasts attenuates renal fibrosis. (A) Schematic diagram of unilateral ureteral obstruction (UUO)-induced renal fibrosis in $M K L 1^{f / f}$ and MKL1 ${ }^{\Delta \mathrm{Fib}}$ mice. Tamoxifen $(50 \mathrm{mg} / \mathrm{kg} / \mathrm{d}$ ) was injected into mice by intraperitoneal injection (i.p.) for 10 days. The mice were then subjected to UUO surgery for 14 days. (B) Masson's trichrome staining. Scale bar, $20 \mu \mathrm{m} . \mathrm{N}=5$ mice. (C) Picrosirius red staining under white light or polarized light. Scale bar, $20 \mu \mathrm{m}$. $\mathrm{N}=5 \mathrm{mice}$. (D) Hydroxylproline level were detected by test kit. $\mathrm{N}=8$ mice. (E) Pro-fibrogenic genes were measured by RT-qPCR. $N=9$ mice. (F) Urine urea nitrogen (BUN) levels. $\mathrm{N}=5$ mice. (G) Urine creatinine $(\mathrm{Cr})$ levels. $\mathrm{N}=5$ mice. Data represent mean $\pm \mathrm{SD}$. ${ }^{\star} p<0.05$, two-tailed $t$-test. 


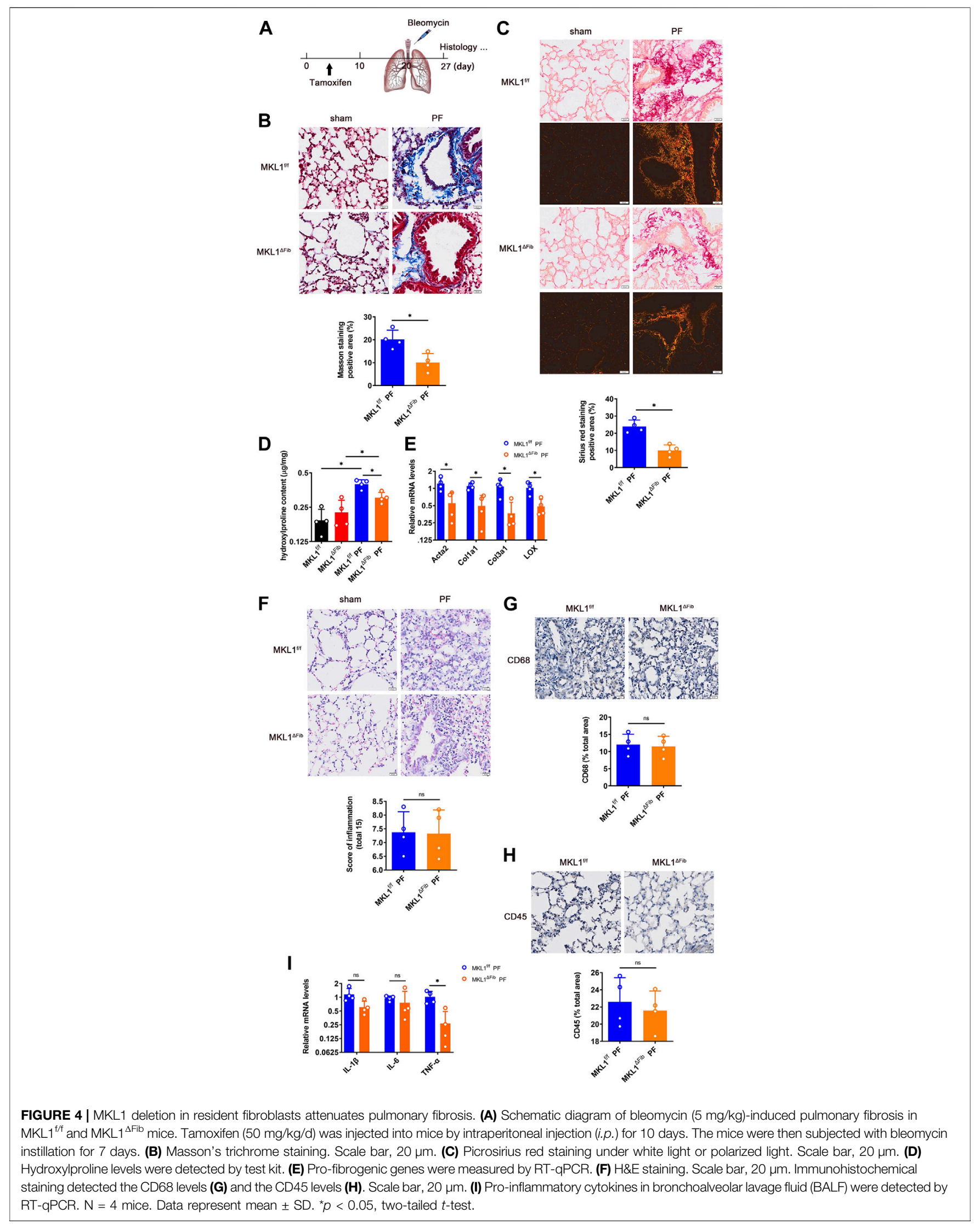


classic model of bleomycin instillation was performed (Figure 4A); typically, an acute inflammatory response takes place in the lungs within 3 days of bleomycin administration, which is followed by interstitial fibrosis (Moeller et al., 2008). Masson's trichrome staining (Figure 4B) and picrosirius red staining (Figure 4C) clearly indicated that interstitial fibrosis was far less extensive in the $M K L 1^{\Delta \mathrm{Fib}}$ lungs than in the $\mathrm{MKL1}^{\mathrm{f} / \mathrm{f}}$ lungs. Figure 4C also showed the down-regulation of collagen fiber size in $\mathrm{MKL}_{1}{ }^{\triangle \mathrm{Fib}}$ lung under polarized light. Quantification of hydroxylproline levels (Figure 4D) and qPCR analysis of profibrogenic gene expression levels (Figure 4E) confirmed an amelioration of pulmonary fibrosis in the $\mathrm{MKL}^{\Delta \mathrm{Fib}}$ mice than in the $\mathrm{MKL1}^{\mathrm{f} / \mathrm{f}}$ mice.

On the other hand, H\&E staining (Figure 4F) indicated that gross pulmonary morphology was not significantly different between the two groups. Immunohistochemical staining performed with an anti-CD68 antibody (Figure 4G), which labels macrophages, and an anti-CD45 antibody (Figure $\mathbf{4 H}$ ), which labels granulocytes, showed similar levels of immune infiltrates in the $\mathrm{MKL1}^{\triangle \mathrm{Fib}}$ lungs and in the $\mathrm{MKL1}^{\mathrm{f} / \mathrm{f}}$ lungs. Finally, when expression of pro-inflammatory cytokines in the cells from the bronchoalveolar lavage fluid (BALF) was examined, it was found that levels of tumor necrosis factor (Tnfa), but not interleukin 1 (Illb) or interleukin 6 (Il6), were lower in the $\mathrm{MKL1}^{\Delta \mathrm{Fib}}$ mice than in the $\mathrm{MKL1}^{\mathrm{f} / \mathrm{f}}$ mice (Figure 4I).

\section{DISCUSSION}

Genetic lineage tracing has enabled the delineation of cellular origins underlying a host of physiological and pathophysiological processes including tissue fibrosis (Baron and van Oudenaarden, 2019). Landmark studies, aided by the lineage tracing tools, have shown that resident fibroblasts are the major or, in some cases, the predominant source from which myofibroblasts arise to mediate the pro-fibrogenic response in various tissues (Sato and Yanagita, 2017; Gibb et al., 2020; Freeberg et al., 2021). MKL1 is a master regulator of tissue fibrosis as evidenced by the observations that global MKL1 deletion or inhibition mitigates fibrosis in the heart (Small et al., 2010; Weng et al., 2015), in the lungs (Zhou et al., 2013; Bernau et al., 2015; Sisson et al., 2015), and in the kidneys (Xu et al., 2015; Bialik et al., 2019; Mao et al., 2020b). Here we present data to show that mice harboring resident fibroblast-specific MKL1 deletion display attenuated tissue fibrosis in several different models suggesting that resident fibroblast MKL1 is sufficient to drive a pro-fibrogenic response in vivo.

We show here that less extensive cardiac fibrosis occurred in the $\mathrm{MKL1}^{\Delta \mathrm{Fib}}$ mice than in the $\mathrm{MKL1}^{\mathrm{f} / \mathrm{f}}$ mice following myocardial infarction. This observation echoes the finding of a previous report by Small et al. that demonstrates an attenuation of cardiac fibrosis in the global MKL1 deficient (KO) mice (Small et al., 2010). Of note, Small et al. reported that global MKL1 deletion improved the post-MI systolic heart function but without statistical significance whereas our data suggest that fibroblast-specific MKL1 deletion significantly elevated postMI systolic function (Figures 2F,G). It is likely MKL1 outside the fibroblast compartments may play a protective role during heart injuries such that the removal of MKL1 from this compartment may neutralize that beneficial effects conferred by deletion of MKL1 in fibroblasts. It is not clear at this point what the identity of this compartment might be. There are many factors that can affect ejection fraction and fractional shortening, such as myocardial microvascular function (Qianwen Zhao. et al., 2019), cardiomyocyte function (Schiattarella et al., 2019), and skeletal muscle mitochondrial function (Anupam A Kumar et al., 2019), the regulation of which MKL1 may not contribute to. Thus, the effect of fibroblast-specific MKL1 deletion on the recovery of ejection fraction and fractional shortening was understandably small. It has been previously shown targeted deletion of MKL1 from either endothelial cells (Weng et al., 2015) or myelocytic cells (Liu et al., 2021a; Liu et al., 2021b) or mature cardiomyocytes (Wu T. et al., 2020) could potentially mitigate the suppression of systolic heart function post injuries. On the other hand, recent studies have indicated that cytoskeletal remodeling, likely in cardiac precursor cells, is essential for heart regeneration post-injury (Morikawa et al., 2015). MKL1 is considered as a master regulator of actin reshuffling in the cells (Olson and Nordheim, 2010). It is plausible to speculate that MKL1 may play a protective role during heart injury by promoting the regenerating potential of precursor cells.

At the initial stage of ligation, one healthy kidney can indeed compensate for the function of the other, injured kidney. However, with the extension of ligation time, this compensatory effect will gradually disappear. Moreover, the glomeruli filtration as evidenced by the measurements of plasma BUN and Cr levels was altered at 2 weeks after UUO (Zhaohui Wang et al., 2020). Our data show that resident fibroblast MKL1 did not significantly alter glomeruli filtration as evidenced by the measurements of plasma BUN and Cr levels. This is because there are many complex factors that affect BUN and Cr levels. Another explanation is the animals in the present study were only observed for 2 weeks following the surgical procedure. Thus, the permanent architectural changes taking place in patients with chronic kidney disease (CKD) or endstage renal disease (ESRD) were not faithfully recapitulated. In contrast, $\mathrm{Xu}$ et al. have previously shown that global MKL1 deletion significantly ameliorated glomeruli function in a model of diabetic nephropathy (Xu et al., 2015). A wealth of data suggests that there is generally an inverse relationship between renal fibrosis and glomerular filtration functions (Djudjaj and Boor, 2019). Therefore, one would expect to record an improvement of glomerular function as a result of reduced fibrosis in the MKL1 ${ }^{\triangle \mathrm{Fib}}$ mice. One explanation why this was not the case could be that the animals in the present study were only observed for 2 weeks following the surgical procedure. Thus, the permanent architectural changes taking place in patients with chronic kidney disease (CKD) or end-stage renal disease (ESRD) were not faithfully recapitulated, which indicates that the UUO model is an imperfect model to study renal fibrosis for it does not encompass the full pathology in humans (Becker and Hewitson, 2013). In a similar vein, it is noteworthy that we did not observe any difference between the MKL1 ${ }^{\Delta \mathrm{Fib}}$ mice and in the $\mathrm{MKL1}^{\mathrm{f} / \mathrm{f}}$ mice in terms of diastolic heart functions (Figures 
$\mathbf{2 H}, \mathbf{I})$ despite the fact that excessive fibrosis tends to increase myocardial rigidity and interferes with the diastolic functions (Burlew and Weber, 2002). Future studies should aim to evaluate the long-term effects of MKL1 deletion in fibroblasts on organ functions.

We have similarly found that fibroblast-specific MKL1 deletion attenuated pulmonary fibrosis but minimally altered the inflammatory response in the lungs. By comparison, an earlier study has found that global MKL1 deletion protects the mice from lipopolysaccharide (LPS) induced pulmonary inflammation in a model of septicemia owing to the regulatory role of MKL1 in macrophages (Yu et al., 2017). However, we did observe a difference in TNF- $\alpha$ expression levels in the cells collected from BALF (Figure 4I). BALF cells mostly are of the immune lineages including macrophages, lymphocytes, and granulocytes (Sterclova et al., 2019). Therefore, the decrease in TNF- $\alpha$ expression could be simply construed as a reduction in immune cell trafficking in the MKL1 ${ }^{\triangle \mathrm{Fib}}$ lungs, which raises an intriguing point that MKL1 in resident fibroblasts is able to regulate not only pro-fibrogenic genes (e.g., collagen) but chemoattractive genes to promote immune cell infiltration. Indeed, pulmonary fibroblasts can function as a source from which chemokines are derived (Boyd et al., 2020). Coincidently, MKL1 has been shown to directly activate the transcription of a slew of chemokines (Yu et al., 2014; Song et al., 2017). Although immunohistochemical staining data indicate that infiltration of neither $\mathrm{CD} 8^{+}$macrophages nor $\mathrm{CD} 45^{+}$granulocytes in the lungs was significantly altered by MKL1 deletion in fibroblasts (Figures $\mathbf{4 G}, \mathbf{H})$, the possibility that specific sets of immune cell populations could be modulated by MKL1-dependent and fibroblast-derived chemokines cannot be completely excluded and deserves further investigation.

In summary, we provide evidence to implicate resident fibroblast derived MKL1 as a sufficient driving force in tissue fibrosis. Despite the clarification that the pro-fibrogenic ability of MKL1 likely emanates from fibroblasts, several outstanding questions remain to be answered. First, the effects of fibroblast-specific MKL1 deletion on tissue fibrosis have been thoroughly investigated through long-term observations because only acute models were employed in the present study. In addition, the conclusion has not been validated in multiple models of tissue fibrosis that typically represent the far complex etiologies in humans. Second, there is a great deal of heterogeneity in fibroblast populations, which is partly determined and shaped by tissue origins (Lynch and Watt, 2018). Although the $M K L 1^{\mathrm{f} / \mathrm{f}}$ mice seem to exhibit similar

\section{REFERENCES}

Baron, C. S., and Van Oudenaarden, A. (2019). Unravelling Cellular Relationships during Development and Regeneration Using Genetic Lineage Tracing. Nat. Rev. Mol. Cel Biol 20, 753-765. doi:10.1038/s41580-019-0186-3

Becker, G. J., and Hewitson, T. D. (2013). Animal Models of Chronic Kidney Disease: Useful but Not Perfect. Nephrol. Dial. Transplant. 28, 2432-2438. doi:10.1093/ndt/gft071 phenotypes in different models of tissue fibrosis, it is unlikely that the transcriptomes of cardiac fibroblasts, pulmonary fibroblasts, and renal fibroblasts might be uniformly altered by the loss of MKL1. Recent advances in single-cell RNAsequencing have greatly facilitated the fine-dissecting the dynamic transcriptomic changes in a wide ranges of tissues and organs (Fu et al., 2021; Liang et al., 2021; Ma et al., 2021; Xie et al., 2021; Zhao et al., 2021). Therefore, a comparison of gene expression profiles of the MKL1-null fibroblasts using this stateof-the-art technique in different organs could shed additional insight on the mode of action for MKL1. These caveats will need to be addressed in future studies so that the field can inch closer to understanding the holistic picture wherein MKL1 contributes to tissue fibrosis.

\section{DATA AVAILABILITY STATEMENT}

The original contributions presented in the study are included in the article/supplementary Material, further inquiries can be directed to the corresponding authors.

\section{ETHICS STATEMENT}

The animal study was reviewed and approved by the intramural Ethics Committee on Humane Treatment of Laboratory Animals of Nanjing Medical University.

\section{AUTHOR CONTRIBUTIONS}

QZ, TL, and XG conceived project; SH, TS, and QZ designed experiments, performed experiments, collected data, and analyzed data; QZ wrote the manuscript; QZ, XG, TL handled funding.

\section{FUNDING}

This work was supported by grants from the National Natural Science Foundation of China (82100286), Natural Science Foundation of the Jiangsu Higher Education Institutions (20KJB310017), and the Key Laboratory of Emergency and Trauma of Ministry of Education (Hainan Medical University) Ministry of Education (KLET-201914).

Bernau, K., Ngam, C., Torr, E. E., Acton, B., Kach, J., Dulin, N. O., et al. (2015) Megakaryoblastic Leukemia-1 Is Required for the Development of BleomycinInduced Pulmonary Fibrosis. Respir. Res. 16, 45. doi:10.1186/s12931-015-0206-6

Bialik, J. F., Ding, M., Speight, P., Dan, Q., Miranda, M. Z., Di Ciano-Oliveira, C., et al. (2019). Profibrotic Epithelial Phenotype: a central Role for MRTF and TAZ. Sci. Rep. 9, 4323. doi:10.1038/s41598-019-40764-7

Boyd, D. F., Allen, E. K., Allen, E. K., Randolph, A. G., Guo, X.-z. J., Weng, Y., et al. (2020). Exuberant Fibroblast Activity Compromises Lung Function via ADAMTS4. Nature 587, 466-471. doi:10.1038/s41586-020-2877-5 
Bujak, M., Kweon, H. J., Chatila, K., Li, N., Taffet, G., and Frangogiannis, N. G. (2008). Aging-related Defects Are Associated with Adverse Cardiac Remodeling in a Mouse Model of Reperfused Myocardial Infarction. J. Am. Coll. Cardiol. 51, 1384-1392. doi:10.1016/j.jacc.2008.01.011

Burlew, B. S., and Weber, K. T. (2002). Cardiac Fibrosis as a Cause of Diastolic Dysfunction. Herz 27, 92-98. doi:10.1007/s00059-002-2354-y

Chevalier, R. L., Forbes, M. S., and Thornhill, B. A. (2009). Ureteral Obstruction as a Model of Renal Interstitial Fibrosis and Obstructive Nephropathy. Kidney Int. 75, 1145-1152. doi:10.1038/ki.2009.86

Cleutjens, J. P., Verluyten, M. J., Smiths, J. F., and Daemen, M. J. (1995). Collagen Remodeling after Myocardial Infarction in the Rat Heart. Am. J. Pathol. 147, 325-338.

Djudjaj, S., and Boor, P. (2019). Cellular and Molecular Mechanisms of Kidney Fibrosis. Mol. Aspects Med. 65, 16-36. doi:10.1016/j.mam.2018.06.002

Fan, Z., Hao, C., Li, M., Dai, X., Qin, H., Li, J., et al. (2015). MKL1 Is an Epigenetic Modulator of TGF- $\beta$ Induced Fibrogenesis. Biochim. Biophys. Acta (Bba) - Gene Regul. Mech. 1849, 1219-1228. doi:10.1016/j.bbagrm.2015.07.013

Freeberg, M. A. T., Perelas, A., Rebman, J. K., Phipps, R. P., Thatcher, T. H., and Sime, P. J. (2021). Mechanical Feed-Forward Loops Contribute to Idiopathic Pulmonary Fibrosis. Am. J. Pathol. 191, 18-25. doi:10.1016/ j.ajpath.2020.09.008

Fu, R., Qin, P., Zou, X., Hu, Z., Hong, N., Wang, Y., et al. (2021). A Comprehensive Characterization of Monoallelic Expression during Hematopoiesis and Leukemogenesis via Single-Cell RNA-Sequencing. Front. Cel Dev. Biol. 9, 702897. doi:10.3389/fcell.2021.702897

Gibb, A. A., Lazaropoulos, M. P., and Elrod, J. W. (2020). Myofibroblasts and Fibrosis. Circ. Res. 127, 427-447. doi:10.1161/circresaha.120.316958

Henderson, N. C., Rieder, F., and Wynn, T. A. (2020). Fibrosis: from Mechanisms to Medicines. Nature 587, 555-566. doi:10.1038/s41586-020-2938-9

Ianni, A., Hofmann, M., Kumari, P., Tarighi, S., Al-Tamari, H. M., Görgens, A., et al. (2021). Depletion of Numb and Numblike in Murine Lung Epithelial Cells Ameliorates Bleomycin-Induced Lung Fibrosis by Inhibiting the $\beta$-Catenin Signaling Pathway. Front. Cel Dev. Biol. 9, 639162. doi:10.3389/ fcell.2021.639162

Johnson, L. A., Rodansky, E. S., Haak, A. J., Larsen, S. D., Neubig, R. R., and Higgins, P. D. R. (2014). Novel Rho/MRTF/SRF Inhibitors Block MatrixStiffness and TGF- $\beta$-Induced Fibrogenesis in Human Colonic Myofibroblasts. Inflamm. Bowel Dis. 20, 154-165. doi:10.1097/ 01.mib.0000437615.98881.31

Kanisicak, O., Khalil, H., Ivey, M. J., Karch, J., Maliken, B. D., Correll, R. N., et al. (2016). Genetic Lineage Tracing Defines Myofibroblast Origin and Function in the Injured Heart. Nat. Commun. 7, 12260. doi:10.1038/ncomms12260

Kaur, H., Takefuji, M., Ngai, C. Y., Carvalho, J., Bayer, J., Wietelmann, A., et al. (2016). Targeted Ablation of Periostin-Expressing Activated Fibroblasts Prevents Adverse Cardiac Remodeling in Mice. Circ. Res. 118, 1906-1917. doi:10.1161/circresaha.116.308643

Klahr, S., and Morrissey, J. (2002). Obstructive Nephropathy and Renal Fibrosis. Am. J. Physiology-Renal Physiol. 283, F861-F875. doi:10.1152/ ajprenal.00362.2001

Kumar, A. A., Kelly, D. P., and Chirinos, J. A. (2019). Mitochondrial Dysfunction in Heart Failure with Preserved Ejection Fraction. Circulation 139, 1435-1450. doi:10.1161/circulationaha.118.036259

Laure, R. (2017). Method for Picrosirius Red-Polarization Detection of Collagen Fibers in Tissue Sections. Methods Mol. Biol. 1627, 395-407.

Lebleu, V. S., Taduri, G., O'connell, J., Teng, Y., Cooke, V. G., Woda, C., et al. (2013). Origin and Function of Myofibroblasts in Kidney Fibrosis. Nat. Med. 19, 1047-1053. doi:10.1038/nm.3218

Li, S., Chang, S., Qi, X., Richardson, J. A., and Olson, E. N. (2006). Requirement of a Myocardin-Related Transcription Factor for Development of Mammary Myoepithelial Cells. Mol. Cel Biol 26, 5797-5808. doi:10.1128/mcb.00211-06

Li, Z., Chen, B., Dong, W., Xu, W., Song, M., Fang, M., et al. (2018). Epigenetic Activation of PERP Transcription by MKL1 Contributes to ROS-Induced Apoptosis in Skeletal Muscle Cells. Biochim. Biophys. Acta Gene Regul. Mech. 27 (18), S187430177-S187493999. doi:10.1016/j.bbagrm.2018.07.011

Liang, J., Shao, W., Liu, Q., Lu, Q., Gu, A., and Jiang, Z. (2021). Single Cell RNASequencing Reveals a Murine Gallbladder Cell Transcriptome Atlas during the Process of Cholesterol Gallstone Formation. Front. Cel Dev. Biol. 9, 714271. doi:10.3389/fcell.2021.714271
Liu, L., Zhao, Q., Kong, M., Mao, L., Yang, Y., and Xu, Y. (2021a). Myocardinrelated Transcription Factor A (MRTF-A) Regulates Integrin Beta 2 Transcription to Promote Macrophage Infiltration and Cardiac Hypertrophy in Mice. Cardiovasc. Res

Liu, L., Zhao, Q., Lin, L., Yang, G., Yu, L., Zhuo, L., et al. (2021b). Myeloid MKL1 Disseminates Cues to Promote Cardiac Hypertrophy in Mice. Front. Cel Dev. Biol. 9, 583492. doi:10.3389/fcell.2021.583492

Lynch, M. D., and Watt, F. M. (2018). Fibroblast Heterogeneity: Implications for Human Disease. J. Clin. Invest. 128, 26-35. doi:10.1172/jci93555

Ma, L., Zhou, N., Zou, R., Shi, W., Luo, Y., Du, N., et al. (2021). Single-Cell RNA Sequencing and Quantitative Proteomics Analysis Elucidate Marker Genes and Molecular Mechanisms in Hypoplastic Left Heart Patients with Heart Failure. Front. Cel Dev. Biol. 9, 617853. doi:10.3389/fcell.2021.617853

Mao, L., Liu, L., Zhang, T., Qin, H., Wu, X., and Xu, Y. (2020a). Histone Deacetylase 11 Contributes to Renal Fibrosis by Repressing KLF15 Transcription. Front. Cel Dev. Biol. 8, 235. doi:10.3389/fcell.2020.00235

Mao, L., Liu, L., Zhang, T., Wu, X., Zhang, T., and Xu, Y. (2020b). MKL1 Mediates TGF- $\beta$-induced CTGF Transcription to Promote Renal Fibrosis. J. Cel Physiol 235, 4790-4803. doi:10.1002/jcp.29356

Meng, X.-M., Wang, S., Huang, X.-R., Yang, C., Xiao, J., Zhang, Y., et al. (2016). Inflammatory Macrophages Can Transdifferentiate into Myofibroblasts during Renal Fibrosis. Cell Death Dis 7, e2495. doi:10.1038/cddis.2016.402

Moeller, A., Ask, K., Warburton, D., Gauldie, J., and Kolb, M. (2008). The Bleomycin Animal Model: a Useful Tool to Investigate Treatment Options for Idiopathic Pulmonary Fibrosis? Int. J. Biochem. Cel Biol. 40, 362-382. doi:10.1016/j.biocel.2007.08.011

Morikawa, Y., Zhang, M., Heallen, T., Leach, J., Tao, G., Xiao, Y., et al. (2015). Actin Cytoskeletal Remodeling with Protrusion Formation Is Essential for Heart Regeneration in Hippo-Deficient Mice. Sci. Signal. 8, ra41. doi:10.1126/ scisignal. 2005781

Olson, E. N., and Nordheim, A. (2010). Linking Actin Dynamics and Gene Transcription to Drive Cellular Motile Functions. Nat. Rev. Mol. Cel Biol 11, 353-365. doi:10.1038/nrm2890

Pakshir, P., Noskovicova, N., Lodyga, M., Son, D. O., Schuster, R., Goodwin, A., et al. (2020). The Myofibroblast at a Glance. J. Cel Sci 133. doi:10.1242/ jcs. 227900

Sato, Y., and Yanagita, M. (2017). Resident Fibroblasts in the Kidney: a Major Driver of Fibrosis and Inflammation. Inflamm. Regener 37, 17. doi:10.1186/ s41232-017-0048-3

Schiattarella, G. G., Altamirano, F., Tong, D., French, K. M., Villalobos, E., Kim, S. Y., et al. (2019). Nitrosative Stress Drives Heart Failure with Preserved Ejection Fraction. Nature 568, 351-356. doi:10.1038/s41586-019-1100-Z

Shiwen, X., Stratton, R., Nikitorowicz-Buniak, J., Ahmed-Abdi, B., Ponticos, M., Denton, C., et al. (2015). A Role of Myocardin Related Transcription Factor-A (MRTF-A) in Scleroderma Related Fibrosis. PLoS One 10-e0126015. doi:10.1371/journal.pone.0126015

Sisson, T. H., Ajayi, I. O., Subbotina, N., Dodi, A. E., Rodansky, E. S., Chibucos, L. N., et al. (2015). Inhibition of Myocardin-Related Transcription Factor/serum Response Factor Signaling Decreases Lung Fibrosis and Promotes Mesenchymal Cell Apoptosis. Am. J. Pathol. 185, 969-986. doi:10.1016/ j.ajpath.2014.12.005

Small, E. M., Thatcher, J. E., Sutherland, L. B., Kinoshita, H., Gerard, R. D., Richardson, J. A., et al. (2010). Myocardin-related Transcription Factor-A Controls Myofibroblast Activation and Fibrosis in Response to Myocardial Infarction. Circ. Res. 107, 294-304. doi:10.1161/circresaha.110.223172

Song, M., Fang, F., Dai, X., Yu, L., Fang, M., and Xu, Y. (2017). MKL1 Is an Epigenetic Mediator of TNF-Alpha-Induced Proinflammatory Transcription in Macrophages by Interacting with ASH2. FEBS Lett

Sterclova, M., Smetakova, M., Stehlik, L., Skibova, J., and Vasakova, M. (2019). Bronchoalveolar Lavage Cell Profiles and Proteins Concentrations Can Be Used to Phenotype Extrinsic Allergic Alveolitis Patients. Multidiscip Respir. Med. 14, 13. doi:10.1186/s40248-019-0175-6

Sun, Y., Boyd, K., Xu, W., Ma, J., Jackson, C. W., Fu, A., et al. (2006). Acute Myeloid Leukemia-Associated Mkl1 (Mrtf-A) Is a Key Regulator of Mammary Gland Function. Mol. Cel Biol 26, 5809-5826. doi:10.1128/mcb.00024-06

Tian, W., Hao, C., Fan, Z., Weng, X., Qin, H., Wu, X., et al. (2015). Myocardin Related Transcription Factor A Programs Epigenetic Activation of Hepatic Stellate Cells. J. Hepatol. 62, 165-174. doi:10.1016/j.jhep.2014.07.029 
Wang, D.-Z., Li, S., Hockemeyer, D., Sutherland, L., Wang, Z., Schratt, G., et al. (2002). Potentiation of Serum Response Factor Activity by a Family of Myocardin-Related Transcription Factors. Proc. Natl. Acad. Sci. 99, 14855-14860. doi:10.1073/pnas.222561499

Wang, L., Zhao, X.-C., Cui, W., Ma, Y.-Q., Ren, H.-L., Zhou, X., et al. (2016). Genetic and Pharmacologic Inhibition of the Chemokine Receptor CXCR2 Prevents Experimental Hypertension and Vascular Dysfunction. Circulation 134, 1353-1368. doi:10.1161/circulationaha.115.020754

Wang, Z., Chen, Z., Li, B., Zhang, B., Du, Y., Liu, Y., et al. (2020). Curcumin Attenuates Renal Interstitial Fibrosis of Obstructive Nephropathy by Suppressing Epithelial-Mesenchymal Transition through Inhibition of the TLR4/NF-Kb and PI3K/AKT Signalling Pathways. Pharm. Biol. 58, 828-837. doi:10.1080/13880209.2020.1809462

Weng, X., Yu, L., Liang, P., Chen, D., Cheng, X., Yang, Y., et al. (2015). Endothelial MRTF-A Mediates Angiotensin II Induced Cardiac Hypertrophy. J. Mol. Cell Cardiol. 80, 23-33. doi:10.1016/j.yjmcc.2014.11.009

Wu, T., Wang, H., Xin, X., Xin, X., Zhang, T., Hou, Y., et al. (2020a). An MRTF-ASp1-PDE5 Axis Mediates Angiotensin-II-Induced Cardiomyocyte Hypertrophy. Front. Cel Dev. Biol. 8, 839. doi:10.3389/fcell.2020.00839

Wu, X., Dong, W., Zhang, T., Ren, H., Wang, J., Shang, L., et al. (2020b). Epiregulin (EREG) and Myocardin Related Transcription Factor A (MRTF-A) Form a Feedforward Loop to Drive Hepatic Stellate Cell Activation. Front Cel Dev Biol 8, 591246. doi:10.3389/fcell.2020.591246

Xie, J., Lou, Q., Zeng, Y., Liang, Y., Xie, S., Xu, Q., et al. (2021). Single-Cell Atlas Reveals Fatty Acid Metabolites Regulate the Functional Heterogeneity of Mesenchymal Stem Cells. Front. Cel Dev. Biol. 9, 653308. doi:10.3389/ fcell.2021.653308

Xu, H., Wu, X., Qin, H., Tian, W., Chen, J., Sun, L., et al. (2015). Myocardin-Related Transcription Factor A Epigenetically Regulates Renal Fibrosis in Diabetic Nephropathy. Jasn 26, 1648-1660. doi:10.1681/asn.2014070678

Xu, T., Lu, Z., Xiao, Z., Liu, F., Chen, Y., Wang, Z., et al. (2020). Myofibroblast Induces Hepatocyte-To-Ductal Metaplasia via Laminin- $\square v \beta 6$ Integrin in Liver Fibrosis. Cel Death Dis 11, 199. doi:10.1038/s41419-020-2372-9

Xu, Y., Luchsinger, L., Lucey, E. C., and Smith, B. D. (2011). The Effect of Class II Transactivator Mutations on Bleomycin-Induced Lung Inflammation and Fibrosis. Am. J. Respir. Cel Mol Biol 44, 898-905. doi:10.1165/rcmb.2009-0416oc

Yang, G., Weng, X., Zhao, Y., Zhang, X., Hu, Y., Dai, X., et al. (2017). The Histone H3K9 Methyltransferase SUV39H Links SIRT1 Repression to Myocardial Infarction. Nat. Commun. 8, 14941. doi:10.1038/ncomms14941

Yu, L., Weng, X., Liang, P., Dai, X., Wu, X., Xu, H., et al. (2014). MRTF-A Mediates LPS-Induced Pro-inflammatory Transcription by Interacting with the COMPASS Complex. J. Cel Sci 127, 4645-4657. doi:10.1242/jcs.152314
Yu, L., Fang, F., Dai, X., Xu, H., Qi, X., Fang, M., et al. (2017). MKL1 Defines the H3K4Me3 Landscape for NF-kB Dependent Inflammatory Response. Sci. Rep. 7, 191. doi:10.1038/s41598-017-00301-w

Zepp, J. A., Zacharias, W. J., Frank, D. B., Cavanaugh, C. A., Zhou, S., Morley, M. P., et al. (2017). Distinct Mesenchymal Lineages and Niches Promote Epithelial Self-Renewal and Myofibrogenesis in the Lung. Cell 170, 1134-1148. doi:10.1016/j.cell.2017.07.034

Zhang, M., Fang, H., Zhou, J., and Herring, B. P. (2007). A Novel Role of Brg1 in the Regulation of SRF/MRTFA-dependent Smooth Muscle-specific Gene Expression. J. Biol. Chem. 282, 25708-25716. doi:10.1074/jbc.m701925200

Zhao, G., Lu, H., Liu, Y., Zhao, Y., Zhu, T., Garcia-Barrio, M. T., et al. (2021) Single-Cell Transcriptomics Reveals Endothelial Plasticity during Diabetic Atherogenesis. Front. Cel Dev. Biol. 9, 689469. doi:10.3389/fcell.2021.689469

Zhao, Q., Yang, J., Chen, H., Li, J., Que, L., Zhu, G., et al. (2019). Pelil Induction Impairs Cardiac Microvascular Endothelium through Hsp90 Dissociation from IRE1a. Biochim. Biophys. Acta (Bba) - Mol. Basis Dis. 1865, 2606-2617. doi:10.1016/j.bbadis.2019.06.017

Zheng, B., Zhang, Z., Black, C. M., De Crombrugghe, B., and Denton, C. P. (2002). Ligand-Dependent Genetic Recombination in Fibroblasts. Am. J. Pathol. 160, 1609-1617. doi:10.1016/s0002-9440(10)61108-x

Zhou, Y., Huang, X., Hecker, L., Kurundkar, D., Kurundkar, A., Liu, H., et al. (2013). Inhibition of Mechanosensitive Signaling in Myofibroblasts Ameliorates Experimental Pulmonary Fibrosis. J. Clin. Invest. 123, 1096-1108. doi:10.1172/jci66700

Conflict of Interest: The authors declare that the research was conducted in the absence of any commercial or financial relationships that could be construed as a potential conflict of interest.

Publisher's Note: All claims expressed in this article are solely those of the authors and do not necessarily represent those of their affiliated organizations, or those of the publisher, the editors, and the reviewers. Any product that may be evaluated in this article, or claim that may be made by its manufacturer, is not guaranteed or endorsed by the publisher.

Copyright (C) 2022 Huang, Shao, Liu, Li, Gui and Zhao. This is an open-access article distributed under the terms of the Creative Commons Attribution License (CC BY). The use, distribution or reproduction in other forums is permitted, provided the original author(s) and the copyright owner(s) are credited and that the original publication in this journal is cited, in accordance with accepted academic practice. No use, distribution or reproduction is permitted which does not comply with these terms. 